\title{
Recovering the Colors of Objects from Multiple Near-IR Images
}

\author{
Ari Kim ${ }^{1}$, In-hoo $\mathrm{Oh}^{1}$, Hong-suk Kim ${ }^{1}$, Seung-ok Park ${ }^{1 *}$, and Youngsik Park ${ }^{2}$ \\ ${ }^{1}$ Department of Physics, Daejin University, Pocheon 487-711, Korea \\ ${ }^{2} 3 D$ Convergence Research Center, Korea Photonics Technology Institute, Gwangju 500-779, Korea
}

(Received November 13, 2014 : revised January 22, 2015 : accepted January 23, 2015)

\begin{abstract}
This paper proposes an algorithm for recovering the colors of objects from multiple near-infrared (near-IR) images. The International Commission on Illumination (CIE) color coordinates of objects are recovered from a series of gray images captured under multiple spectral near-IR illuminations using polynomial regression. The feasibility of the proposed algorithm is tested experimentally by using 24 color patches of the Color Rendition Chart. The experimental apparatus is composed of a monochrome digital camera without an IR cut-off filter and a custom-designed LED illuminator emitting multiple spectral near-IR illuminations, with peak wavelengths near the red edge of the visible band, namely at 700, 740, 780 , and $860 \mathrm{~nm}$. The average color difference between the original and the recovered colors for all 24 patches was found to be 11.1. However, if some particular patches with high value are disregarded, the average color difference is reduced to 4.2, and this value is within the acceptability tolerance for complex image on the display.
\end{abstract}

Keywords : Recovering colors of objects, Night vision, Near-IR image, Multiple spectral near-IR illuminations, The red edge of the visible band

OCIS codes : (330.0330) Vision, color, and visual optics; (330.1690) Color; (100.2000) Digital image processing; (100.2980) Image enhancement

\section{INTRODUCTION}

The human eye is typically capable of detecting light at wavelengths between 400 and $700 \mathrm{~nm}$, a range known as the visible band. The human eye contains photoreceptors with three kinds of color-sensitive pigments for absorbing energy in this wavelength range, thereby enabling the eyes to see [1]. Without the presence of light, the colors as well as the shapes of objects in the scene being observed would disappear.

The development of infrared imaging technology has provided us with night vision, which is the ability to see in conditions of low light or total darkness. Although night vision devices were previously only used by military forces, they have since become more widely available for civilian use. Night vision technologies can be divided into three main categories: low-light imaging, thermal imaging and nar-IR illumination, each of which has its advantages and disadvantages. A popular and sometimes inexpensive technique for achieving night vision uses a device that is sensitive to invisible near-IR radiation in conjunction with a near-IR illuminator. This technique can be performed by most cameras, because their sensors can detect near-IR wavelengths. However, the resulting scene appears as a monochrome image containing single-color or false-color tones which do not correspond to everyday experiences $[2,3]$. Therefore, humans experience monochrome images as highly unnatural.

Furthermore, in situations in which it is critical to obtain color information, such as for accurate identification or tracking purposes, a color image is much more advantageous than a monochrome image. Over the past 20 years, much research has been devoted to producing true-color images from near-IR imaging. The main focus of these studies involves the fusion of visible and infrared images, a technique for which the results are promising when the corresponding day-time images are available [4-9]. Meanwhile, attempts to produce color images by using only infrared light have led to the development of various applications in which a set of three sensors that are individually sensitive to different wavelengths in the near-IR band are used. For example, a

\footnotetext{
*Corresponding author: sopark@daejin.ac.kr

Color versions of one or more of the figures in this paper are available online.
} 
new night vision camera with a set of three near-IR sensors was recently developed by AIST (the National Institute of Advanced Industrial Science and Technology in Japan) [10]. It has been reported that this camera can record clear, high-frame-rate color videos even in darkness, and can reproduce colors that are equivalent or similar to the colors of the same objects under visible light. However, even though the assessment data for the color accuracy has not yet been released, the examples presented by AIST indicate that an improvement in the color signal processing method is required.

This paper proposes an algorithm to recover the colors of objects from multiple near-IR images. The key point is to use the information from the reflectance curve in the wavelength range from 700 to $900 \mathrm{~nm}$, which is near the red edge of the visible band. In Section II, the proposed algorithm is described with the aid of a flowchart. The International Commission on Illumination (CIE) color coordinates $L^{*}, a^{*}, b^{*}$ of the colors of objects are recovered from a series of gray images captured under multiple spectral near-IR illuminations. In Section III, the optical properties of the experimental components, such as the spectral power distribution and spatial uniformity of a custom-designed illuminator and the spectral reflectance of the target color objects, are described in detail. In Section IV, the experimental results that were obtained by analyzing the 24 patches of the Color Rendition Chart [11] are presented. The accuracy of the proposed algorithm is evaluated using the average color difference $\Delta E_{a b}^{*}$. Finally, conclusions and possibilities for further studies are given in Section V.

\section{COLOR RECOVERY ALGORITHM}

Figure 1 shows an example containing the reflectance curves for four color patches painted with red, green, yellow, and blue paint, respectively. According to the definitions that are used in night vision technology [12], the visible band is considered to include wavelengths ranging from

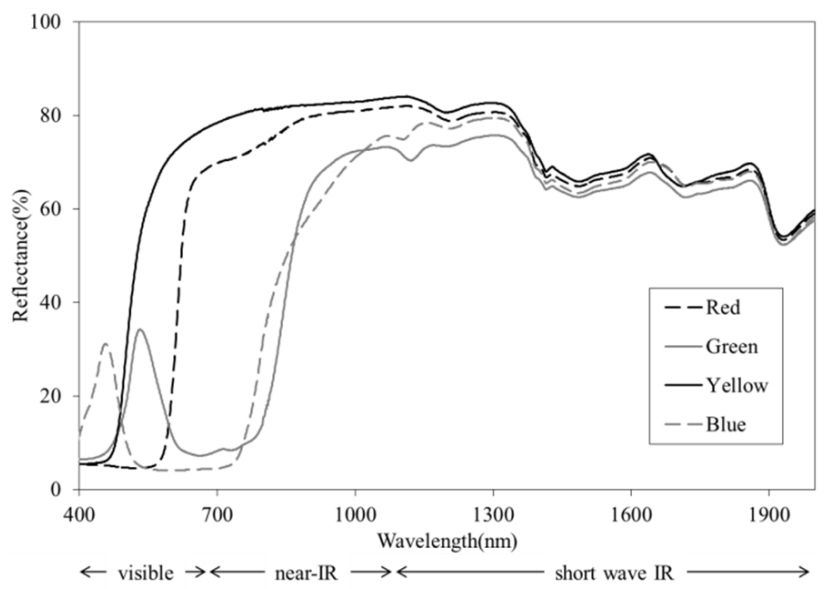

FIG. 1. Reflectance curves for 4 color patches painted with red, green, yellow and blue paint.
400 to $700 \mathrm{~nm}$, the near-IR region is defined to include wavelengths between 700 and $1100 \mathrm{~nm}$, whereas IR wavelengths longer than $1100 \mathrm{~nm}$ are defined as the shortwave IR band that is useful for the detection of objects in a scene.

Figure 1 presents several noteworthy features. The reflectance curve in the visible band has a particular structure that depends on color. However, in the near-IR band, the reflectance curves of the four colors tend to converge to gradually become identical throughout the shortwave IR band. This behavior has been observed for many different materials [13].

The reflectance curve in the visible band is particularly important since it uniquely describes the color of the object as perceived by human observers. In 1931, the CIE created the CIE $X, Y, Z$ color space based on the three photoreceptors in the eye [14]. The $X, Y, Z$ coordinates are defined as the tristimulus values in the range of wavelengths from 380 to $780 \mathrm{~nm}$, as follows;

$$
\begin{aligned}
& X=\int_{380}^{780} P(\lambda) R_{V}(\lambda) \bar{x}(\lambda) d \lambda \\
& Y=\int_{380}^{780} P(\lambda) R_{V}(\lambda) \bar{y}(\lambda) d \lambda \\
& Z=\int_{380}^{780} P(\lambda) R_{V}(\lambda) \bar{z}(\lambda) d \lambda
\end{aligned}
$$

where, $\lambda$ is the wavelength measured in nanometers, $\mathrm{P}(\lambda)$ is the spectral power distribution of light illumination, $R_{\nu}(\lambda)$ is the reflectance of the object in the visible band, and $\bar{x}(\lambda)$, $\bar{y}(\lambda), \bar{z}(\lambda)$ are the color matching functions for the standard observer. These numerical descriptions may be considered the spectral sensitivity curves of the three linear light detectors. In Eq. (1), $\bar{x}(\lambda), \bar{y}(\lambda), \bar{z}(\lambda)$ are approximately zero for wavelengths below $400 \mathrm{~nm}$ and above $700 \mathrm{~nm}$.

In 1976 the CIE $L^{*}, a^{*}, b^{*}$ color space, which is currently used as a perceptually uniform color space, was derived from the CIE $X, Y, Z$ color space [14]. In the new color space $L^{*}$ represents the lightness, $a^{*}$ denotes the red/green value, and $b^{*}$ the yellow/blue value. The non-linear transformation from $X, Y, Z$ to $L^{*}, a^{*}, b^{*}$ is as follows;

$$
\begin{aligned}
& L^{*}=\left\{\begin{array}{l}
116\left(\frac{Y}{Y_{n}}\right)^{\frac{1}{3}}-16 \text { if } \frac{Y}{Y_{n}}>0.008856 \\
903.3\left(\frac{Y}{Y_{n}}\right) \quad \text { if } \frac{Y}{Y_{n}} \leq 0.008856
\end{array}\right. \\
& a^{*}=500 \times\left(f\left(X / X_{n}\right)-f\left(Y / Y_{n}\right)\right) \\
& b^{*}=200 \times\left(f\left(\frac{Y}{Y_{n}}\right)-f\left(\frac{Z}{Z_{n}}\right)\right) \\
& \text { where } f(t)=\left\{\begin{array}{cc}
t^{\frac{1}{3}} & \text { if } t>0.008856 \\
7.787 \times t+16 / 116 & \text { if } t \leq 0.008856
\end{array}\right.
\end{aligned}
$$


where $X_{n}, Y_{n}, Z_{n}$ are the $X, Y, Z$ of the reference white. $\boldsymbol{L}^{*}$ $=0$ yields black and $\boldsymbol{L}^{*}=100$ indicates diffuse white. Along the $a^{*}$ axis, positive values indicate the amount of red, while negative values indicate the amount of green. Similarly, along the $b^{*}$ axis, positive and negative values are indicative of yellow and blue, respectively. For both axes, zero is neutral gray. The advantage of the CIE $L^{*} a^{*} b^{*}$ color space is that the color differences between two colors can be expressed in as follows;

$$
\Delta E_{a b}^{*}=\sqrt{\left(\Delta L^{*}\right)^{2}+\left(\Delta a^{*}\right)^{2}+\left(\Delta b^{*}\right)^{2}}
$$

The reflectance curve in the near-IR band does not contribute to defining the color in terms of $X, Y, Z$ or $L^{*}$, $a^{*}, b^{*}$ since the near-IR band is outside of the visible band. However, for an object under near-IR illumination with wavelengths between 700 and $1100 \mathrm{~nm}, N I R_{1}, N I R_{2}$, $N_{I} R_{3}$ corresponding to $X, Y, Z$ can be represented by the equivalent formula:

$$
\begin{aligned}
& N I R_{1}=\int_{700}^{1100} P(\lambda) R_{N I R}(\lambda) \operatorname{nir}_{1}(\lambda) d \lambda \\
& N I R_{2}=\int_{700}^{1100} P(\lambda) R_{N I R}(\lambda) \operatorname{nir}_{2}(\lambda) d \lambda \\
& N I R_{3}=\int_{700}^{1100} P(\lambda) R_{N I R}(\lambda) \operatorname{nir}_{3}(\lambda) d \lambda
\end{aligned}
$$

where, $\mathrm{P}(\lambda)$ is the spectral power distribution of a near-IR illumination, $R_{N I R}(\lambda)$ is the reflectance of the object in the near-IR band, and $\operatorname{nir}_{1}(\lambda), \operatorname{nir}_{2}(\lambda), \operatorname{nir}_{3}(\lambda)$ are the spectral sensitivity curves of the three near-IR sensors, which can be thought of as the equivalent of $\bar{x}(\lambda), \bar{y}(\lambda), \bar{z}(\lambda)$ in Eq. (1). Alternatively, Eq. (4) can be reformulated to include three spectral near-IR illuminators and a sensor that responds to wavelengths in the near-IR band.

$$
\begin{aligned}
& N I R_{1}=\int_{700}^{1100} P_{1}(\lambda) R_{N I R}(\lambda) S(\lambda) d \lambda \\
& N I R_{2}=\int_{700}^{1100} P_{2}(\lambda) R_{N I R}(\lambda) S(\lambda) d \lambda \\
& N I R_{3}=\int_{700}^{1100} P_{3}(\lambda) R_{N I R}(\lambda) S(\lambda) d \lambda
\end{aligned}
$$

where $P_{1}(\lambda), P_{2}(\lambda)$, and $P_{3}(\lambda)$ are the spectral power distributions of three spectral near-IR illuminators, with the subscript symbolizing the peak wavelength, and $S(\lambda)$ is the spectral sensitivity curve of the near-IR sensor. Most charge- coupled devices (CCDs) or CMOS devices respond to wavelengths ranging from visible to near-IR. $N I R_{1}, N I R_{2}, N I R_{3}$ in Eq. (4) represent the tristimulus values, which are sequentially captured under three spectral near-IR illumination.

Before the proposed recovery algorithm is presented, a simple combination commonly used in remote sensing applications is considered [15]. The values $N I R_{1}, N I R_{2}, N I R_{3}$ are assigned to the $\mathrm{R}, \mathrm{G}$, and B primary signals, respectively, for the purpose of reproducing a color image. These values may occur in many possible combinations depending on the order in which they occur or on their weighting factors. However, the resulting product for any combination is known as a false-color image that does not bear any resemblance to its original color.

The aim of the proposed algorithm is to recover the CIE color coordinates of objects from a series of gray images captured under three spectral near-IR illuminations. This can be achieved using the polynomial regression of the form [16]

$$
\vec{V}_{C I E}=C M M \cdot \vec{V}_{N I R}
$$

where $\vec{V}_{C I E}$ and $\vec{V}_{N I R}$ are the vector forms of the CIE color coordinates $X, Y, Z$ or $L^{*}, a^{*}, b^{*}$ and the near-IR tristimulus values $N I R_{1}, N I R_{2}, N I R_{3}$, respectively. The color mapping matrix $C M M$ represents the numerical relationship between $\vec{V}_{C I E}$ and $\vec{V}_{N I R}$. For a set of target colors with known CIE color coordinates, a CMM is easily computed from the near-IR tristimulus value $N I R_{1}, N I R_{2}, N I R_{3}$ using MATLAB with the formula

$$
C M M=\vec{V}_{C I E} / \vec{V}_{N I R}
$$

The accuracy of $C M M$ depends on the degree of correlation between $\vec{V}_{C I E}$ and $\vec{V}_{N I R}$ of the target colors. However, the aspects of the reflectance curves in the visible band $R_{V}(\lambda)$ and in the near-IR band $R_{N I R}(\lambda)$ are quite different as shown in Fig. 1; therefore, the correlation between $\vec{V}_{C I E}$ and $\vec{V}_{N I R}$ would be expected to be low.

To improve the recovery performance, the proposed algorithm is designed to acquire an individual $C M M$ for each group consisting of highly correlated colors that are selected from a set of target colors. Figure 2 shows the flowchart of the color recovery process from $N I R_{1}, N I R_{2}, N I R_{3}$ to $L^{*}$, $a^{*}, b^{*}$.

In order to classify the highly correlated colors together, a new set of parameters is derived from the near-IR tristimulus value $N I R_{1}, N I R_{2}, N I R_{3}$ as follows;

$$
\begin{aligned}
& I_{S}=N I R_{1}+N I R_{2}+N I R_{3} \\
& G_{21}=N I R_{2}-N I R_{1} \\
& G_{32}=N I R_{3}-N I R_{2}
\end{aligned}
$$




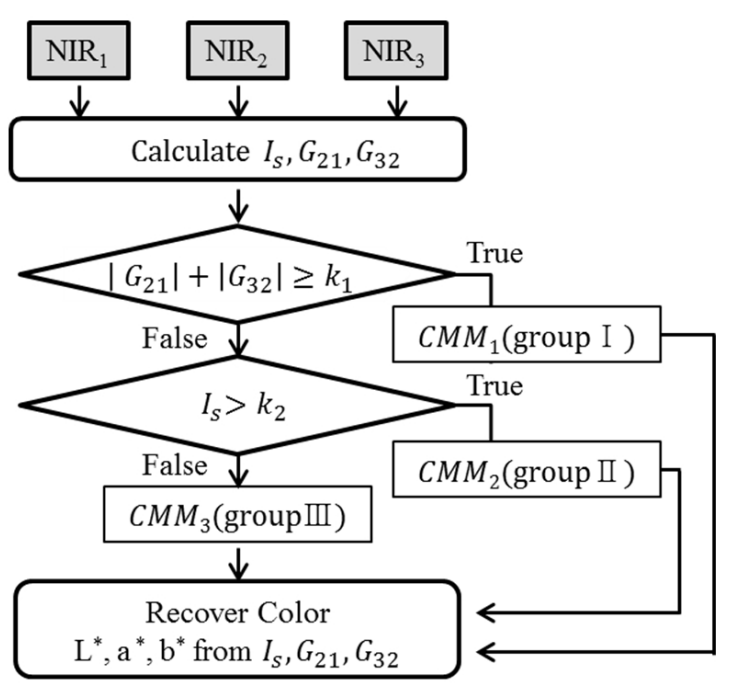

FIG. 2. Flowchart of color recovery process from $N I R_{1}, N I R_{2}$, $N I R_{3}$ to $L^{*}, a^{*}, b^{*}$.

In Eq. (8), $I_{\mathrm{s}}$ means the height of the reflectance curve $R_{N I R}(\lambda)$. While $G_{21}$ and $G_{32}$ mean the gradient of the reflectance curve $R_{N I R}(\lambda)$ between adjacent wavebands, respectively. For both $G_{21}$ and $G_{32}$, positive values indicate an increase in the reflectance with increasing wavelength, whereas negative values indicate a decreasing reflectance.

As can be seen in Fig. 1, selected reflectance curves in the near-IR band exhibit steep gradients, while others display gentle gradients. In this study, each set of target colors is classified into two groups based on the gradient of the reflectance curve, following which the gentle gradient group is additionally classified into two further groups based on the height of the reflectance curve. The workflow shown in Fig. 2 consists of three paths. If the sum of $\left|G_{21}\right|+\left|G_{32}\right|$ of a target color is greater than or equal to a threshold value $k_{1}$ then it belongs to group I. If not, if the value of $I_{S}$ is determined to be greater than a threshold value $k_{2}$, the color is considered to belong to group II, otherwise it belongs to group III. The threshold values $k_{1}$ and $k_{2}$ are properly determined according to constituent target colors. The target colors assigned to each group using this method are highly correlated with each other. The individual color mapping matrices $C M M_{1}, C M M_{2}, C M M_{3}$ can be computed for separate groups using Eq. (7). Accordingly, for colors belonging to the same group, the values of $L^{*}$, $a^{*}, b^{*}$ can be recovered as follows:

$$
\vec{V}_{C I E}=C M M \cdot \vec{V}_{I G G}
$$

where $\vec{V}_{C I E}$ and $\vec{V}_{I G G}$ are the vector forms of $L^{*}, a^{*}, b^{*}$ and $I_{\mathrm{s}}, G_{21}, G_{32}$, respectively. $C M M$ represents the individual color mapping matrix of the group. The performance of the proposed algorithm is evaluated by determining the average color difference $\Delta E^{*}{ }_{a b}$ between the original and recovered values.

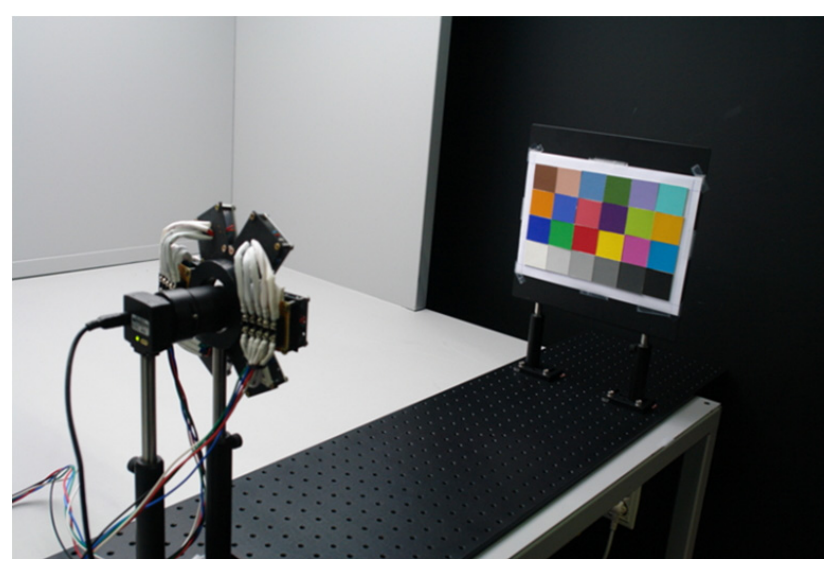

FIG. 3. Experimental configuration.

\section{EXPERIMENTAL SETUP}

The experiment is carried out in dark condition. The experimental room (including the ceiling and the wall) is painted matte black. The experimental configuration is presented in Fig. 3. A screen that is attached to the target color objects is placed $0.7 \mathrm{~m}$ from a multiple spectral near-IR illuminator. Through the hole in the center of the illuminator, a monochrome digital camera is placed facing the screen as shown in Fig. 3. The camera has an image sensor consisting of a diagonal 1/3 inch CCD array with $1.25 \mathrm{M}$ effective pixels. Its spectral response ranges from 300 to $1000 \mathrm{~nm}$, and the maximum sensitivity is near $580 \mathrm{~nm}$. Using the camera's software, automatic white balance function and a gamma value are set to 'off' and 1, respectively. The 16-bit data of the captured image is saved in TIFF format.

Details of the illuminator and target color objects are described in the following sections.

\subsection{Multiple Spectral Near-IR Illuminator}

The multiple spectral near-IR illuminator consisting of six near-IR LED array modules is custom-designed. Figure 4 (a) shows a schematic diagram of one of the six modules. In the diagram, (A), B), C), and (D) indicate four types of near-IR LEDs with peak wavelengths at 700, 740, 780 and $860 \mathrm{~nm}$, respectively. Each module consists of a uniform arrangement of four of each of these types, i.e., a total of 16 LEDs per module. Figure 4 (b) shows the assembled multiple spectral near-IR illuminator with the six modules, which are slightly tilted toward the central hole in the form of a sunflower. The luminous intensity of the LEDs in all six modules is controlled according to type by using four separate power supplies. For the experiment, the three types of LEDs with peak wavelengths at 700, 780 and 860 $\mathrm{nm}$ were selected.

Figure 5 presents the spectral power distributions of these three types of LEDs as measured by using a USB-650 spectrometer. In Fig. 5, $P(\lambda)_{700}, P(\lambda)_{780}$ and $P(\lambda)_{860}$ denote the spectral power distributions of the three types of LEDs, 


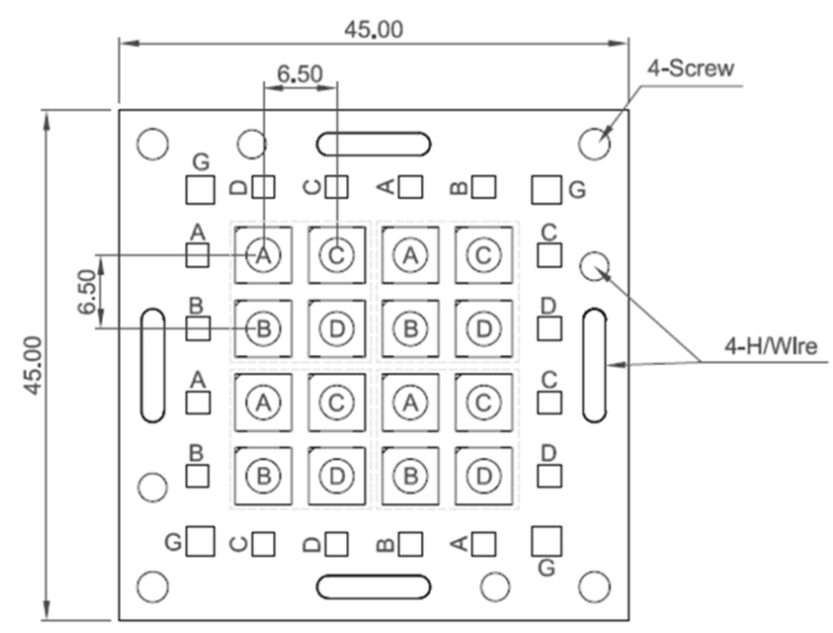

(a)

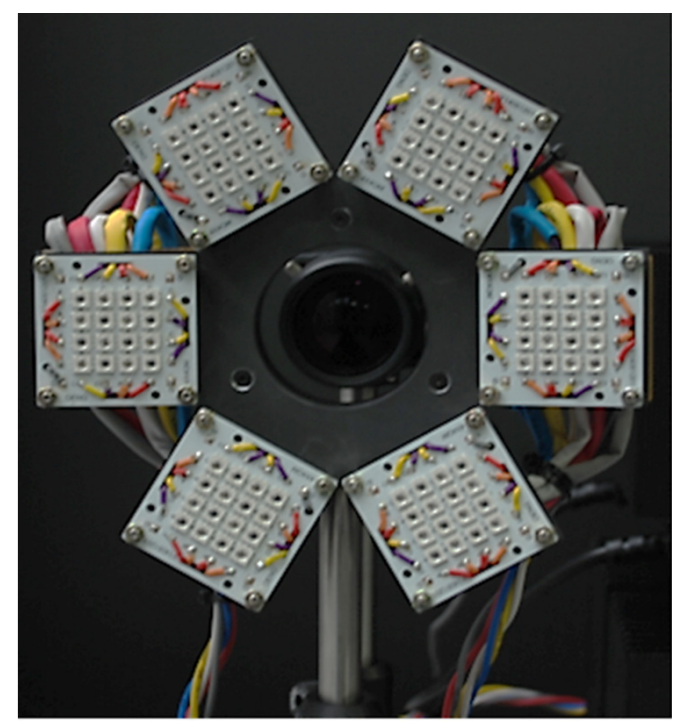

(b)

FIG. 4. (a) Schematic diagram for one of 6 modules and (b) an assembled multiple spectral near-IR illuminator with six modules.

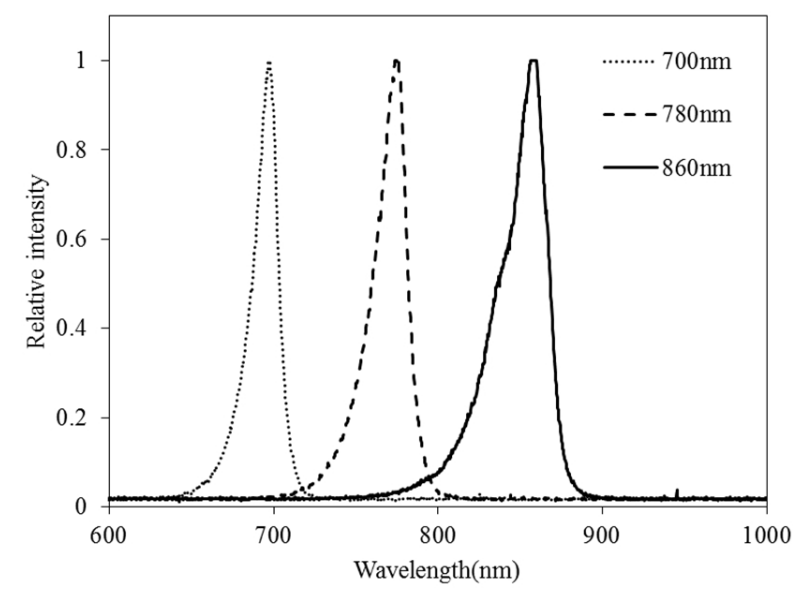

FIG. 5. The spectral power distributions of the three types of LEDs; 700, 780 and $860 \mathrm{~nm}$. of which the respective maximum values are normalized to 1 .

The spatial uniformity of the custom-designed illuminator was measured by using a uniform gray card (X-rite ColorChecker Custom White Balance Card, of which the size of the square, excluding the border, is $27.4 \times 17.8$ $\mathrm{cm})$. In Figure 3, the white balance card is attached to the screen. Figure 6 shows the captured images of the white balance card. The uniformity of the entire plane is shown at a glance in Fig. 6 (a), (b), and (c), in which the resultant color-coded images that were obtained under the illumination of $P(\lambda)_{700}, P(\lambda)_{780}$ and $P(\lambda)_{860}$, respectively, are shown. The strength of the input current of each of the

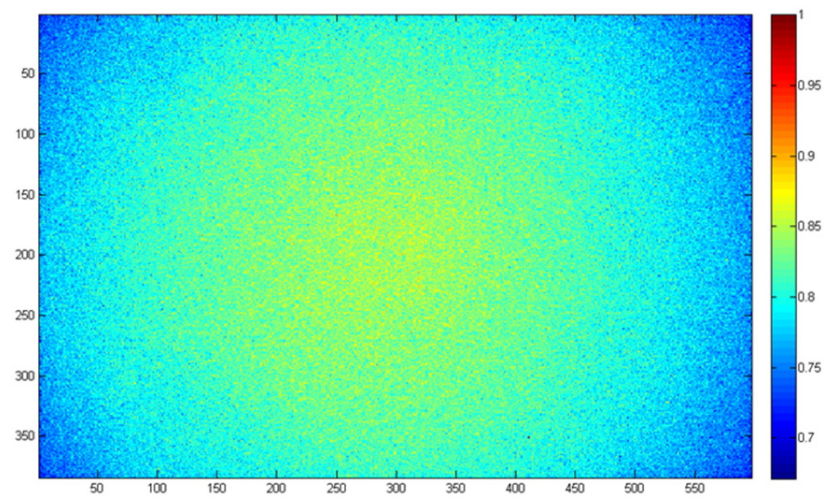

(a)

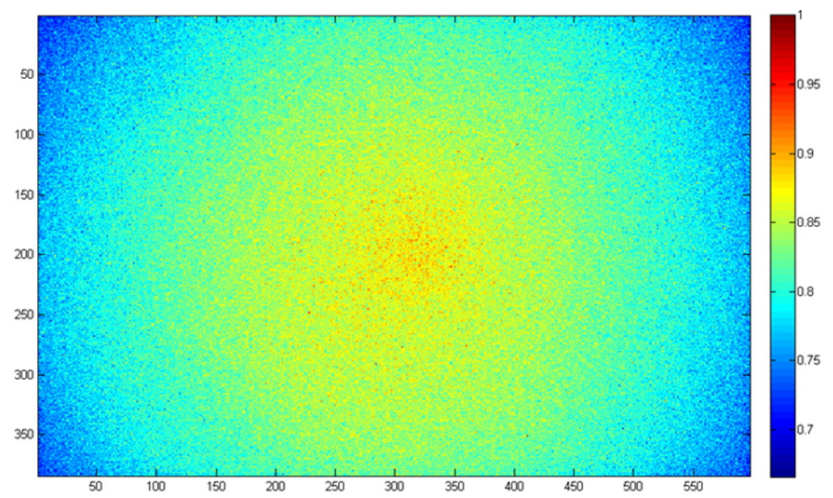

(b)

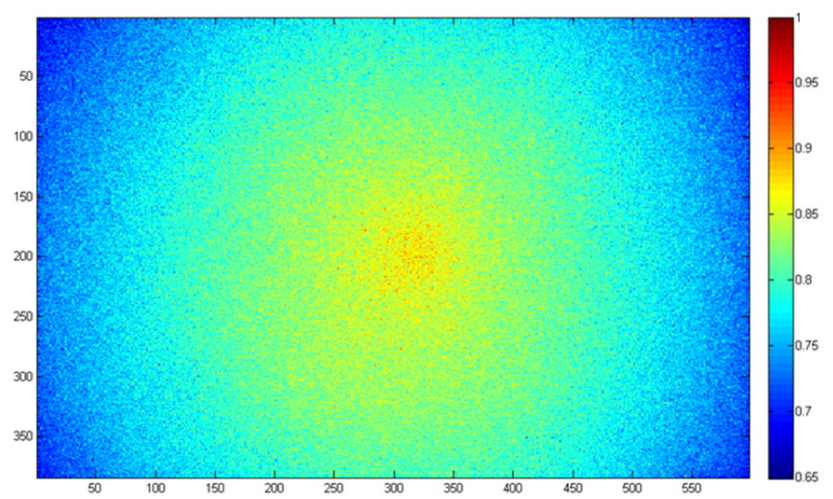

(c)

FIG. 6. The captured images of the white balance card under the three types of LEDs; (a) $700 \mathrm{~nm}$ (b) $780 \mathrm{~nm}$ (c) $860 \mathrm{~nm}$. 
three types of LEDs was independently adjusted to equalize the brightness of their respective images, a process referred to as adjusting the white balance.

In addition, the pixel positions of each of the images in Fig. 6 correspond to the size of the gray card $(27.4 \times 17.8$ $\mathrm{cm})$. All three of the color-coded images display concentric rings of which the color gradually changes, and in which the maximum code value is 1 near the center. Toward the edges of the gray card, the code value is gradually reduced to about 0.7 in the center of the $\mathrm{x}$-axis and 0.8 in the center of the y-axis. Comparing the three images in Fig. 6, the degree of non-uniformity of $P(\lambda)_{700}, P(\lambda)_{780}$ and $P(\lambda)_{860}$ can be seen to vary slightly from one image to another. The effects of the non-uniformity of the illumination were minimized by multiplying each pixel data in the captured image by the value corresponding to the inverse of the code value shown in Fig. 6.

\subsection{Target Color Objects}

The ColorChecker is generally used as a color calibration target in visible imaging technology. It includes 24 color patches in a $6 \times 4$ grid, each slightly under 2 inches square, made of matte paint applied to smooth paper, and surrounded by a black border. A photograph of the ColorChecker is shown in Fig. 7, in which the original colors as they appear in visible light are shown. Six of the patches form a uniform gray lightness scale; white (w), neutral 8 (n8), neutral 6.5 (n6.5), neutral 5 (n5), neutral 3.5 (n3.5), and black (bk). Another six patches represent the primary colors; red (r), green (g), blue (b), cyan (c), magenta $(\mathrm{m})$, and yellow $(\mathrm{y})$. The remaining colors include approximations of natural colors, such as human skin tones (ds and ls), blue sky (bs), a typical leaf (f), a blue chicory flower (bf), and bluish green (bg). The remaining colors were chosen arbitrarily to represent a gamut: orange (o), purplish blue (pb), moderate red (md), purple (p), yellow green (yg), and orange yellow (oy). The letters in parentheses represent the acronym for each tone.

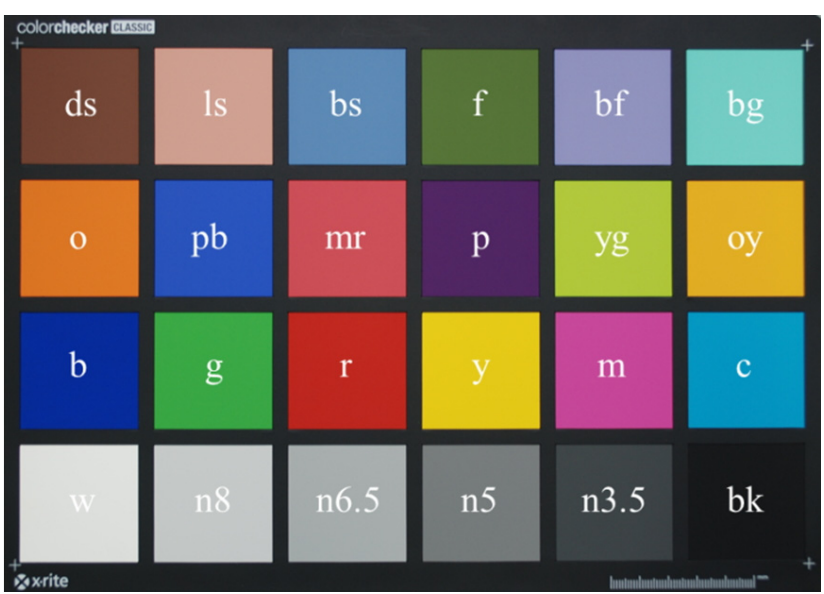

FIG. 7. The photograph of ColorChecker captured under visible light.
The spectral reflectance of each patch was measured by using a Cary 5000 UV-Vis-NIR spectrophotometer after cutting the 24 patches from the ColorChecker. Figure 8 shows the accumulated reflectance curve for all 24 patches in the wavelength range from 400 to $1100 \mathrm{~nm}$, which includes both the visible and the near-IR bands. As expected, based on the explanation in Fig. 1, the reflectance data shows the inherent structure of each color in the visible band $(400-700 \mathrm{~nm})$. In the near-IR band, the reflectance data increases near the red edge of the visible band (700 900 $\mathrm{nm})$ before assuming a uniform appearance. As the red

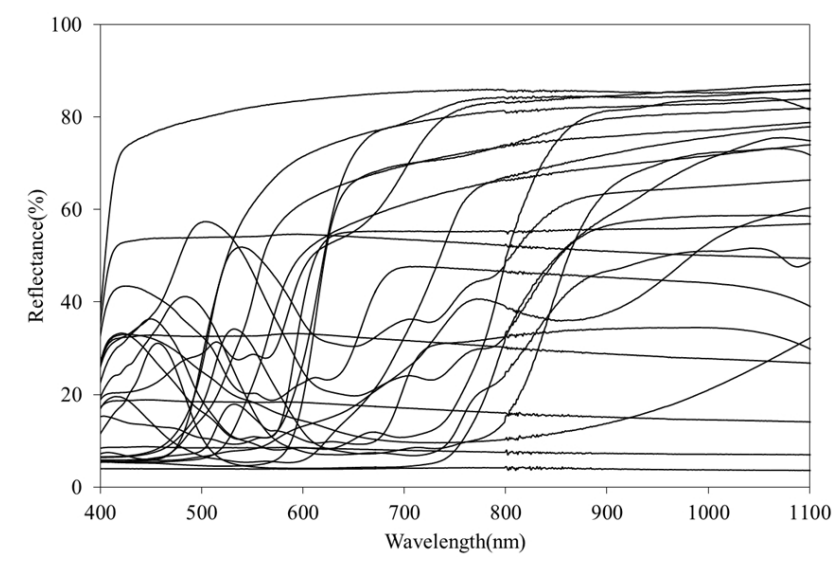

FIG. 8. The reflectance curve for all 24 patches in the wavelength range from 400 to $1100 \mathrm{~nm}$.

TABLE 1 . The $X, Y, Z$ values for Illuminants $D_{65}$ and the derived $L^{*}, a^{*}, b^{*}$ values of 24 patches

\begin{tabular}{c|l|c|c|c|c|c|c}
\hline \multirow{2}{*}{ No. } & \multirow{2}{*}{ Number } & \multicolumn{3}{|c|}{ CIE XYZ } & \multicolumn{3}{c}{ CIE Lab } \\
\cline { 3 - 8 } & & $\mathrm{X}$ & $\mathrm{Y}$ & $\mathrm{Z}$ & $\mathrm{L}^{*}$ & $\mathrm{a}^{*}$ & $\mathrm{~b}^{*}$ \\
\hline 1 & dark skin(ds) & 12.5 & 11.3 & 7.8 & 40.1 & 12.2 & 13.7 \\
\hline 2 & light skin(1s) & 42.3 & 39.5 & 29.6 & 69.1 & 15.0 & 17.1 \\
\hline 3 & blue sky(bs) & 20.1 & 21.5 & 39.3 & 53.4 & -1.5 & -22.7 \\
\hline 4 & foliage(f) & 11.6 & 14.9 & 8.3 & 45.5 & -16.8 & 21.3 \\
\hline 5 & blue flower(bf) & 28.9 & 27.4 & 51.0 & 59.3 & 11.8 & -25.5 \\
\hline 6 & bluish green(bg) & 35.4 & 48.5 & 51.3 & 75.2 & -33.4 & 1.6 \\
\hline 7 & orange(o) & 40.6 & 32.7 & 7.2 & 63.9 & 32.1 & 57.0 \\
\hline 8 & purplish blue(pb) & 15.0 & 13.1 & 40.9 & 43.0 & 15.8 & -42.7 \\
\hline 9 & moderate red(mr) & 31.5 & 21.7 & 16.6 & 53.7 & 45.5 & 13.3 \\
\hline 10 & purple(p) & 9.9 & 7.6 & 16.6 & 33.2 & 22.9 & -22.3 \\
\hline 11 & yellow green(yg) & 37.1 & 48.6 & 13.2 & 75.2 & -27.8 & 58.3 \\
\hline 12 & orange yellow(oy) & 52.8 & 49.3 & 9.6 & 75.6 & 16.0 & 68.9 \\
\hline 13 & blue(b) & 9.2 & 7.0 & 31.3 & 31.7 & 23.7 & -49.7 \\
\hline 14 & green(g) & 16.3 & 26.2 & 11.4 & 58.3 & -42.1 & 33.7 \\
\hline 15 & red(r) & 23.1 & 13.6 & 6.5 & 43.7 & 54.6 & 24.8 \\
\hline 16 & yellow(y) & 63.6 & 67.4 & 10.9 & 85.7 & -0.9 & 82.3 \\
\hline 17 & magenta(m) & 33.0 & 22.1 & 33.8 & 54.1 & 49.4 & -14.6 \\
\hline 18 & cyan(c) & 15.9 & 21.5 & 42.5 & 53.5 & -24.2 & -26.3 \\
\hline 19 & white(w) & 90.9 & 95.9 & 96.9 & 98.4 & -0.5 & 4.9 \\
\hline 20 & neutral8(n8) & 60.2 & 63.4 & 68.0 & 83.7 & -0.1 & 0.9 \\
\hline 21 & neutral6.5(n6.5) & 36.5 & 38.3 & 41.5 & 68.3 & 0.2 & 0.3 \\
\hline 22 & neutral5(n5) & 20.3 & 21.4 & 23.8 & 51.6 & 0.0 & 0.0 \\
\hline 23 & neutral3.5(n3.5) & 9.4 & 9.9 & 11.0 & 36.2 & 0.0 & 0.0 \\
\hline 24 & black(bk) & 4.4 & 4.6 & 5.0 & 20.3 & 0.0 & 0.0 \\
\hline & & & & & & \\
\hline
\end{tabular}


edge of the visible band is approached (Fig. 8), the reflectance data of some colors increase rapidly and their reflectance curve displays a steep gradient, whereas other colors have a gentler gradient. Using the measured spectral reflectance data in the visible band, it is possible to calculate the $X$, $Y, Z$ values for Illuminants $D_{65}$ using Eq. (1) and also to derive the $L^{*}, a^{*}, b^{*}$ values using Eq. (2). The results are presented in Table 1.

As shown in the experimental setup, the 24 cut patches were rearranged in the form of a $6 \times 4$ grid without the black borders to prepare them for use as target color objects. The target object was created to have the same size as the gray card that is shown in Fig. 6 to compensate for non-uniformity in illumination.

\section{RESULTS}

Figure 9 shows a set of monochrome images, after compensation for non-uniformity, that was experimentally obtained from the target color objects. Figure 9 (a), (b), and (c) show the results of illumination at $P(\lambda)_{700}, P(\lambda)_{780}$ and $P(\lambda)_{860}$ respectively. The input current of each of the three types of LEDs was individually adjusted to achieve white balance. The longer the wavelength of the illumination, the more intense the brightness observed for the image. This tendency is consistent with the trend displayed by the reflectance curves shown in Figure 8 . As a result of the effect of the white balance, the brightness of a series of neutral patches in the bottom row of the target color objects is invariant to any illumination.

Figure 9 was used to define the set of tristimulus values $P(\lambda)_{700}, P(\lambda)_{780}$ and $P(\lambda)_{860}$ for the target color objects. These values correspond to the normalized pixel data of the images in Fig. 9 (a), (b), and (c), respectively, because the gamma function of the camera was set to 1 . Figure 10 shows examples of composite images that were composed using a simple combination method. Figure 10 (a) is formed by assigning the values of $P(\lambda)_{700}, P(\lambda)_{780}$ and $P(\lambda)_{860}$, to the $\mathrm{R}, \mathrm{G}$, and $\mathrm{B}$ primary signals, respectively. Figure 10 (b) and (c) are similarly formed by assigning these values to $G, B$, and $R$, and $B, R$, and $G$, respectively. Figure 10 (a), (b), and (c) show monotones of green, blue, and red, respectively, i.e., they do not bear any resemblance to the original color.

Figure 11 shows a color image that was recovered from the three near-IR images in Fig. 9 by using the proposed algorithm. The 24 patches are classified into three groups on the basis of the criteria suggested by the proposed algorithm using MATLAB. Figure 12 (a), (b), and (c) illustrate the reflectance curves from 700 to $900 \mathrm{~nm}$ of the color patches belonging to groups I, II, and III, respectively. The bluish or greenish patches, all of which have a steep gradient, are included in group I, whereas the reddish or yellowish patches, which have a gentle gradient and a large reflectance sum, are included in group II. The

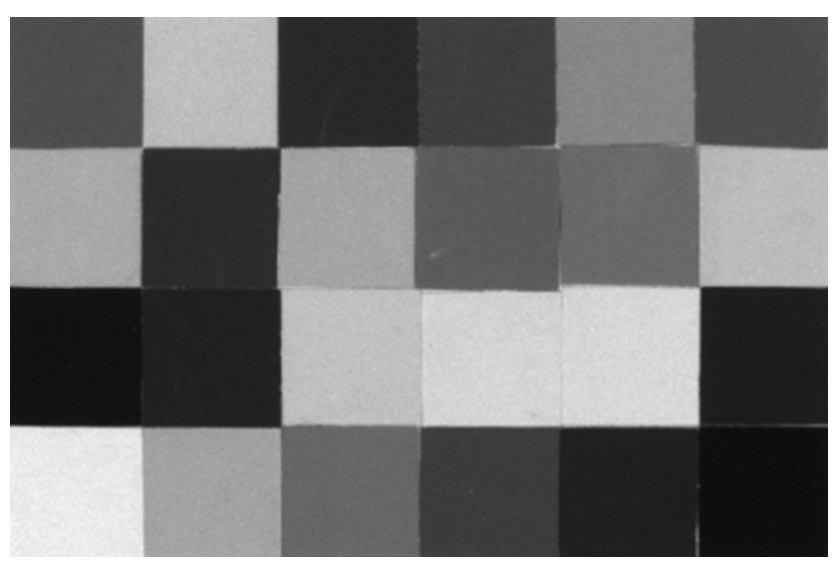

(a)

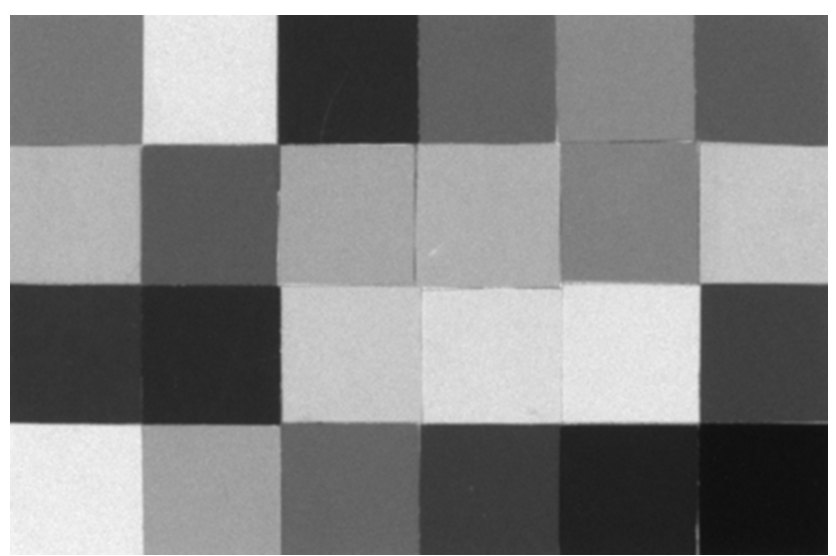

(b)

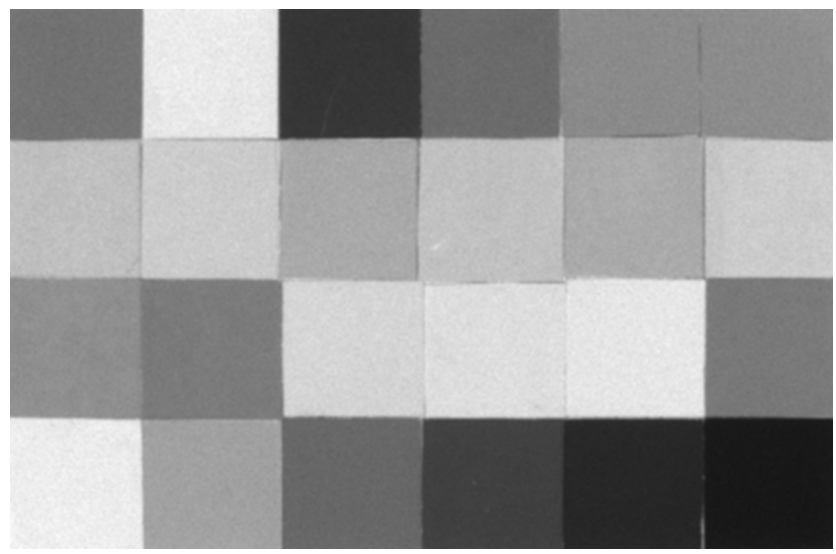

(c)

FIG. 9. A set of monochrome images of the target color objects obtained experimentally with the illumination of (a) $P(\lambda)_{700}$, (b) $P(\lambda)_{780}$ and (c) $P(\lambda)_{860}$.

remaining patches, which have a gentle gradient and a small reflectance sum, are included in group III. The individual color mapping matrix $C M M_{1}, C M M_{2}$, and $C M M_{3}$ was subsequently computed for all three of these groups. The noise level was suppressed by applying a polynomial transformation model with $3 \times 20$ terms [17] to all pixels constituting each patch. By using $C M M_{1}$, $C M M_{2}$, and $C M M_{3}$, the $L^{*}, a^{*}, b^{*}$ values of all 24 patches 


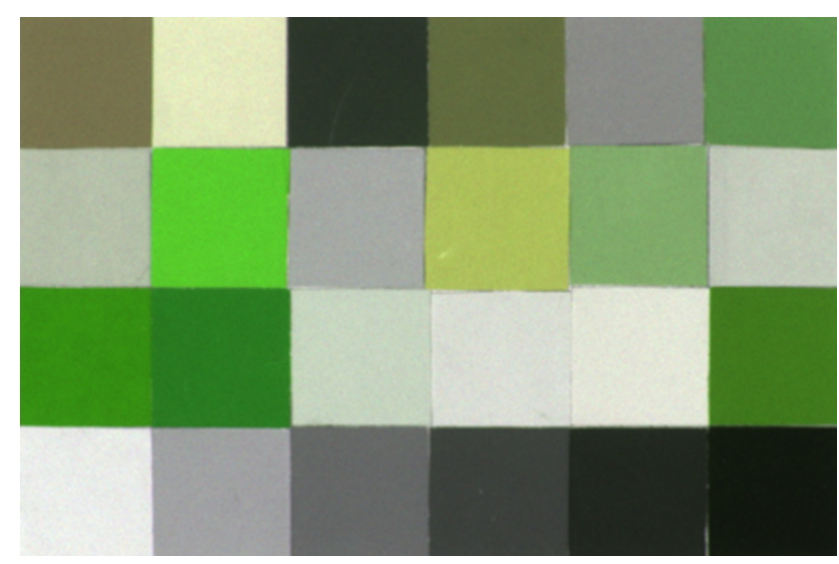

(a)

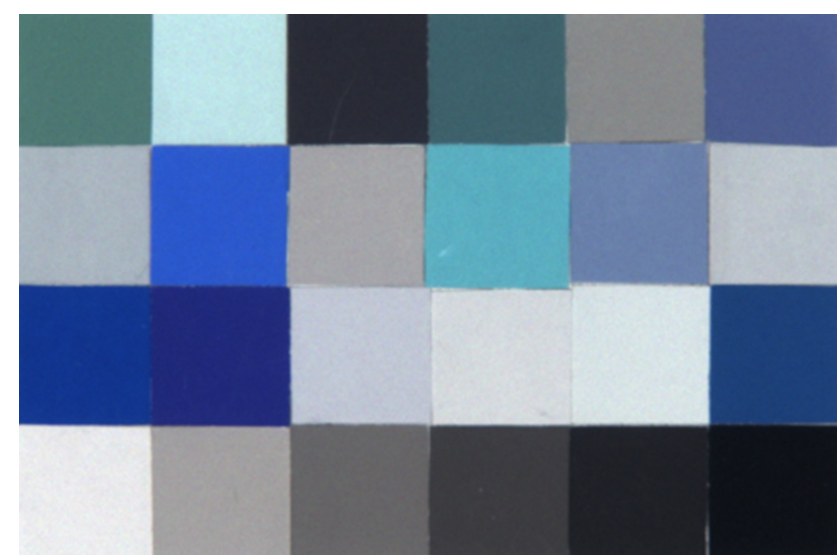

(b)

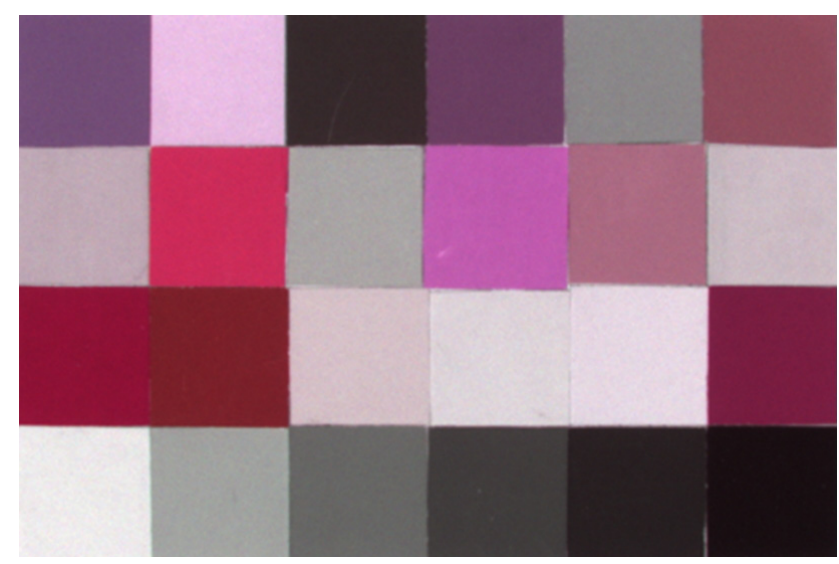

(c)

FIG. 10. Examples of composite image using simple combination method; $N I R_{700}, N I R_{780}, N I R_{860}$ are assigned to (a) (R, G and B), (b) (G, B and R) or (c) (B, R, and G).

were recovered by using Eq. (9). Figure 11 shows a sRGB color image obtained by transforming the recovered $L^{*}$, $a^{*}, b^{*}$ values to sRGB using MATLAB [18].

At a glance, the colors in Fig. 11 appear very similar to those of the original patches. However, some colors differ significantly from the original. The color difference $\Delta E^{*}{ }_{a b}$ between the calculated (Table 1) and the recovered values of each patch is calculated using Eq. (3) and shown in

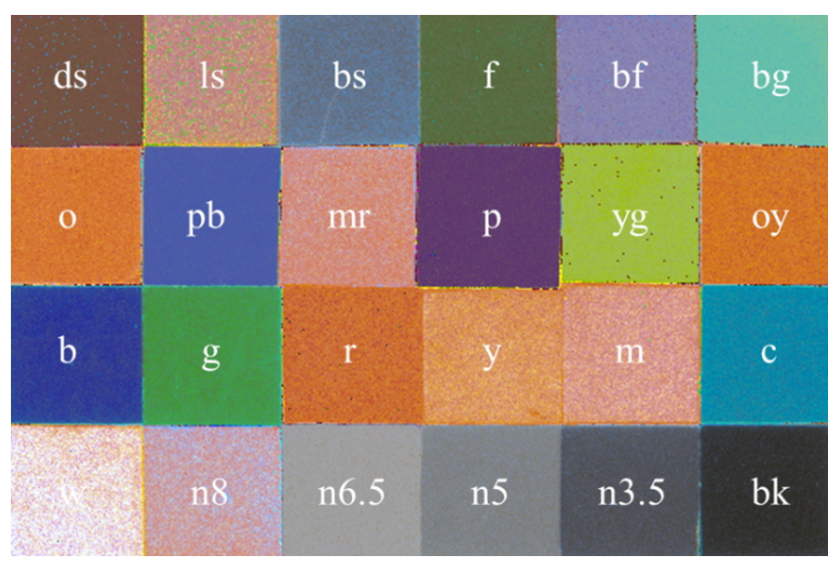

FIG. 11. A color image recovered from the three near-IR images using the proposed algorithm.

TABLE 2. The color difference between original and recovered colors

\begin{tabular}{|c|c|c|c|}
\hline \multirow{2}{*}{ Group } & \multirow{2}{*}{ Name } & \multicolumn{2}{|c|}{ Average per } \\
\hline & & Patch & Group \\
\hline \multirow{7}{*}{ I } & bg & 2.6 & \multirow{7}{*}{3.5} \\
\hline & $\mathrm{pb}$ & 1.1 & \\
\hline & $\mathrm{p}$ & 1.7 & \\
\hline & yg & 4.1 & \\
\hline & $\mathrm{b}$ & 5.0 & \\
\hline & $\mathrm{g}$ & 5.5 & \\
\hline & $\mathrm{c}$ & 4.5 & \\
\hline \multirow{9}{*}{ II } & ls & 9.6 & \multirow{9}{*}{25.9} \\
\hline & o & 7.3 & \\
\hline & $\mathrm{mr}$ & 25.8 & \\
\hline & oy & 24.5 & \\
\hline & $\mathrm{r}$ & 33.5 & \\
\hline & $\mathrm{y}$ & 47.9 & \\
\hline & $\mathrm{m}$ & 47.4 & \\
\hline & $\mathrm{W}$ & 15.4 & \\
\hline & n8 & 21.8 & \\
\hline \multirow{8}{*}{ III } & ds & 2.6 & \multirow{8}{*}{3.8} \\
\hline & bs & 8.0 & \\
\hline & $\mathrm{f}$ & 3.1 & \\
\hline & bf & 2.9 & \\
\hline & $\mathrm{n} 6.5$ & 1.8 & \\
\hline & n5 & 3.9 & \\
\hline & $\mathrm{n} 3.5$ & 5.0 & \\
\hline & bk & 2.9 & \\
\hline
\end{tabular}

Table 2. The average $\Delta E^{*}{ }_{a b}$ across each group is also shown in Table 2. The average $\Delta E^{*}{ }_{a b}$ for the patches in groups I and III are 3.5 and 3.8, respectively. On the other hand, the average $\Delta E^{*}{ }_{a b}$ for the patches in group II is 25.9, and then this value is extremely high compared with the results of other groups. The color difference for each 


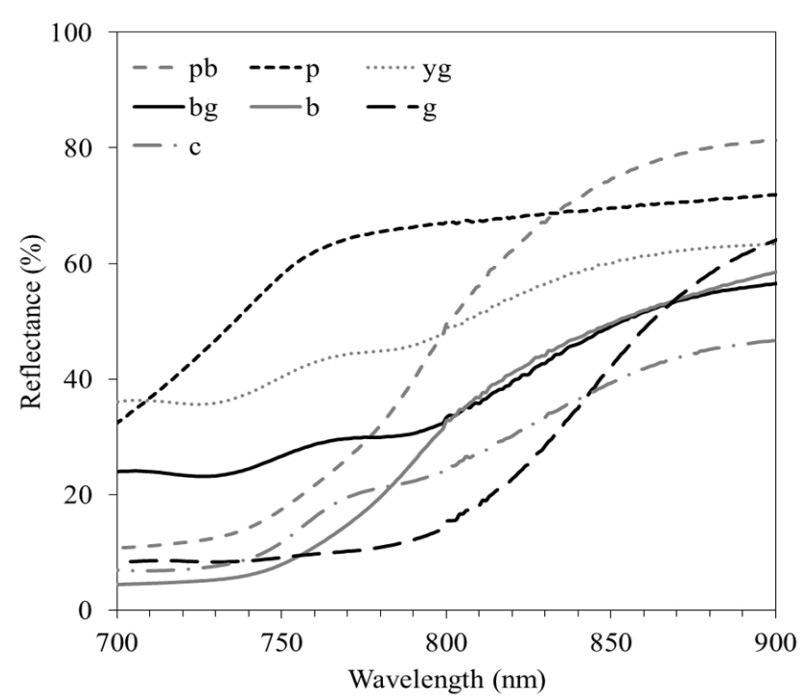

(a)

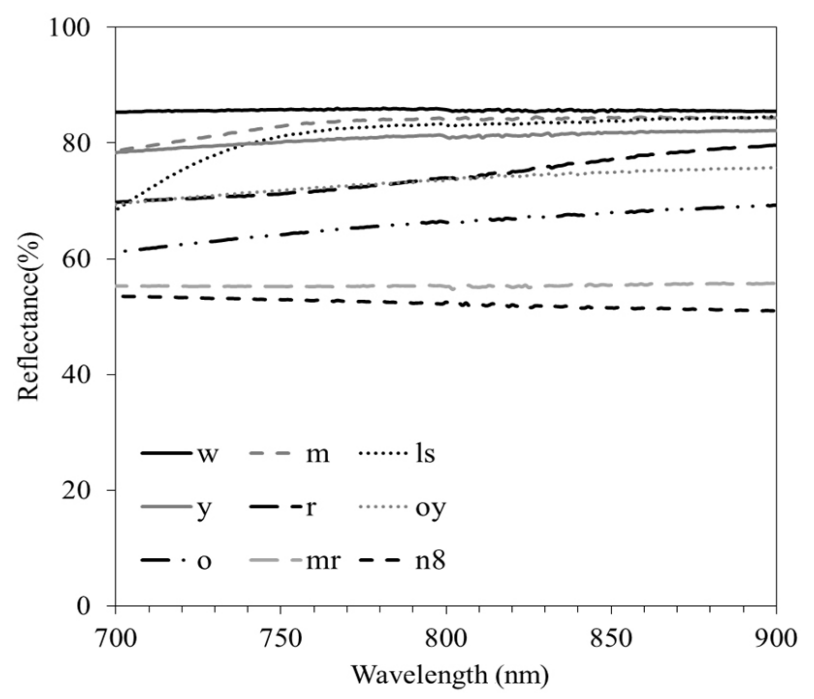

(b)

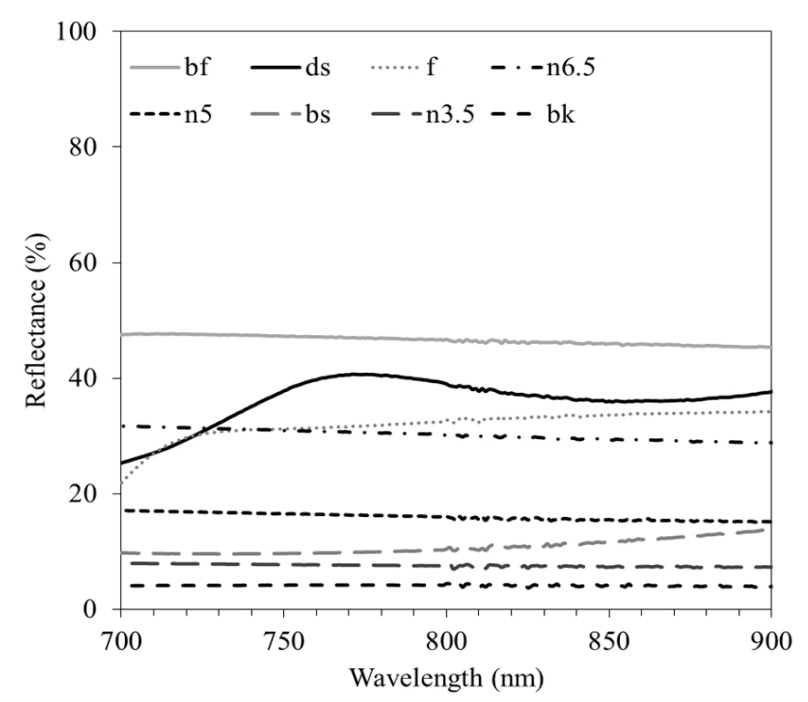

(c)

FIG. 12. The reflectance curves of target color objects belong in each group; (a) group I, (b) group II and (c) group III. patch is analyzed in the lightness difference $\Delta L^{*}$ and the chromaticity shift in the $a^{*} b^{*}$ plane in Fig. 13 (a) and (b), respectively. The results for the seven colors in group II $(\mathrm{mr}$, oy, $\mathrm{r}, \mathrm{y}, \mathrm{m}, \mathrm{w}$, and $\mathrm{n} 8$ ) show comparatively large values for both the lightness difference in Fig. 13 (a) and the chromaticity shift in Fig. 13 (b). It is interesting that for the lightness difference shown in Fig. 13 (a), one pair of colors consisting of $\mathrm{mr}$ and $\mathrm{n} 8$ shows a similar value of opposite sign, a result that was also found for another pair consisting of oy and $r$. And a set of three colors; $y$, $\mathrm{w}$ and $\mathrm{m}$ show the similar result. These results were observed, because the reflectance curves in the wavelength range from 700 to $900 \mathrm{~nm}$ of each of these pairs are almost indistinguishable as can be seen in Fig. 12 (b).

The average color difference $\Delta E^{*}{ }_{a b}$ for all 24 patches is 11.1. However, if the patches of which the reflectance curves are indistinguishable in group II are disregarded, the average color difference $\Delta E^{*}{ }_{a b}$ is reduced to 4.2 , and this value is within the acceptability tolerance for complex image on the display $[19,20]$.

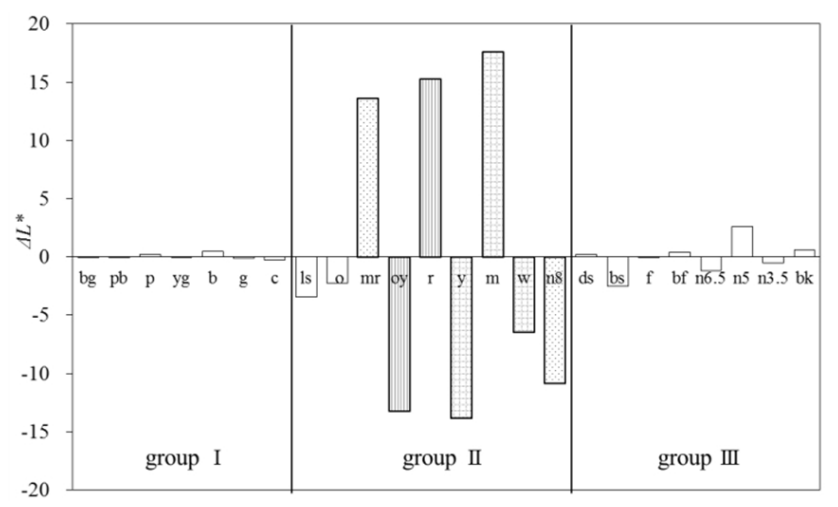

(a)

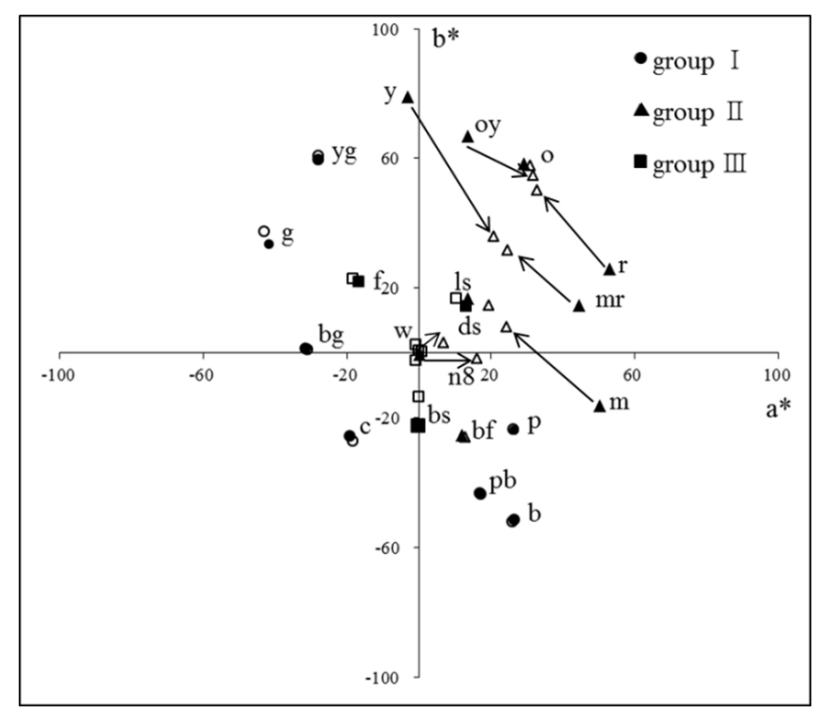

(b)

FIG. 13. (a) Color difference in the lightness $\Delta L$ and (b) the chromaticity shift in the $a^{*} b^{*}$ plane. 


\section{CONCLUSION}

An algorithm to recover the colors of objects from multiple near-IR images is proposed. The CIE color coordinates $L^{*}$, $a^{*}, b^{*}$ of the objects are recovered from a series of gray images captured under multiple spectral near-IR illuminations using the polynomial regression. The feasibility of the proposed algorithm is confirmed experimentally using the 24 color patches of the ColorChecker. The average color difference that was obtained for all 24 patches is 11.1 in $\Delta E^{*}{ }_{a b}$ unit. If seven patches with high value are disregarded, the average color difference $\Delta E^{*}{ }_{a b}$ is reduced to 4.2 , and this value is within the acceptability tolerance for complex image on the display.

In the next study, a feasibility test will be continued for an extended number of color patches using the other Color Rendition Chart, for example 169 colors made of matte paint of the ColorChecker DC. Near-IR reflectance behavior of artifacts and natural objects made of various materials also should be investigated in further research. In addition, the spatial uniformity of the illuminator could be enhanced by improving the optical design of components, such as the lens, and the beam shaping diffuser [21]. It is expected that multiple-spectral illuminations provided by an illuminator with a narrow half-width, such as a laser, used in conjunction with a high-resolution camera, would be helpful to enhance the color image quality.

\section{ACKNOWLEDGMENT}

The authors would like to thank Sung Ok Won, Senior Research Scientist of Korea Institute of Science and Technology (KIST) for his help in measuring the spectral reflectance of target color patches by using the Cary 5000 UV-Vis-NIR Spectrophotometer.

\section{REFERENCES}

1. D. L. Schacter, D. T. Gilbert, and D. M. Wegner, Psychology (Worth Publishers, New York, USA, 2009).

2. M. Vilaseca, J. Pujol, M. Arjona, and F. M. M. Verdu, "Color visualization system for near-Infrared multispectral images," in Proc. 2nd CGIV (Penang, Malaysia, Apr. 2004), pp. 431-436.

3. M. Vilaseca, J. M. de Lasarte, J. Pujol, M. Arjona, and F. M. M. Verdu, "Multispectral system for the reflectance reconstruction and color visualization of natural and manufactured objects in the near-infrared region," in Proc. AIC Colour 10th Congress (Granada, Spain, May 2005), pp. 499-502.

4. V. Tsagaris and V. Anastassopoulos, "Fusion of visible and infrared imagery for night color vision," Displays 26, 191-196 (2005).

5. J. Han and B. Bhanu, "Fusion of color and infrared video for moving human detection," J. Elsevier Pattern Recognition 40, 1771-1784 (2007).

6. X. Qian, Y. Wang, and B. Wang, "Effective contrast enhancement method for color night vision," J. Elsevier Infrared Phys. \& Tech. 55, 130-136 (2012).

7. Z. Chen, X. Wang, and R. Liang, "RGB-NIR multispectral camera," J. Opt. Soc. America 22, $4985-4994$ (2014).

8. A. Toet, "Natural colour mapping for multiband ngihtvision imager," J. Info. Fusion 4, 155-166 (2003).

9. S. R. Dastjerdi, M. Ghanaatshoar, and T. Hattori, "Nearinfrared subwavelength imaging and focusing analysis of a square lattice photonic crystal made from partitioned cylinders," J. Opt. Soc. Korea 17, 262-268 (2013).

10. Y. Nagamune, "Image capturing device and image capturing method," Korea Patent 10-1381018 (2014)

11. C. S. McCamy, H. Marcus, and J. G. Davidson, "A color-rendition chart," J. Appl. Photographic Engineering 2, 95-99 (1976).

12. A. Richards, Alien Vision (SPIE, Bellingham, Washington, USA, 2011).

13. T. Haran, "Short-wave infrared diffuse reflectance of textile materials," in Physics and Astronomy Theses (Academic, Georgia State University Press, Georgia, USA, 2008).

14. R. S. Berns, Billmeyer and Saltzman's Principles of Color Technology (Wiley-Interscience, 2000).

15. T. Lillesand, R. W. Kiefer, and J. Chipman, Remote Sensing and Image Interpretation, 6th ed. (Wiley, USA, 2007).

16. H. R. King, Computational Color Technology (SPIE, USA, 2006).

17. Y. J. Kim and M. R. Luo, "Characterization of a LCD colour monitor using a digital still camera," in Proc. AIC Colour 10th Congress (Granada, Spain, May 2005), pp. 295-298.

18. S. Westland, C. Ripamonti, and V. Cheung, Computational Colour Science Using MATLAB, 2nd ed. (Wiley, Chennai, India, 2012).

19. A. Kim, H. S. Kim, and S. O. Park, "Measuring of the perceptibility and acceptability in various color quality measures," J. Opt. Soc. Korea 15, 310-317 (2011).

20. D. H. Kim, H. S. Kim, S. O. Park, and Y. J. Kim, "Perceptual quality of still images," in Proc. 25th Session of the CIE (San Diego, USA, Jul. 2003), pp. 8-14.

21. B. Y. Joo and J. H. Ko, "Analysis of color uniformity of white LED lens packages for direct-lit LED backlight applications,” J. Opt. Soc. Korea 17, 506-512 (2013). 